\title{
Estimación del riesgo por movimientos en masa en la subcuenca del río Ancash - Yungay
}

Estimation of risk by mass movements in the sub-basin of the Ancash river - Yungay

${ }^{1}$ Jhonior Tarazona Mendoza ${ }^{\mathrm{a}},{ }^{1}$ Rosa Rodríguez Anaya ${ }^{\mathrm{a}},{ }^{1}$ Henrry Garrido Angulo ${ }^{\mathrm{b}}$

\section{RESUMEN}

La subcuenca del río Ancash ubicado al este de la ciudad de Yungay, tiene características físicamente muy variadas expresadas en aspectos geológicos, geomorfológicos e hidrológicos. El aumento en la frecuencia e intensidad de los fenómenos climáticos extremos, producto de la variación en las condiciones atmosféricas que se conoce como cambio climático, ha desencadenado la ocurrencia de eventos tales como movimientos en masa. No existen muchos trabajos en el Perú con relación al análisis de los factores detonantes (pendiente, geología, geomorfología y uso de tierras) y desencadenantes (precipitación) para evaluar los riesgos por movimientos en masa con enfoque de cuenca, la mayoría de estos trabajos se ha realizado en cuencas experimentales bien instrumentadas, pero muy pocos de estos estudios se ha realizado tomando las características climáticas como las anomalías en la precipitación siendo uno de los variables el fenómeno "El Niño" que genera impactos desastrosos a la población y sus medios de vida. El estudio se realizó con la metodología de "Proceso de Análisis Jerárquico" (PAJ) que permitió medir los criterios cuantitativos y cualitativos mediante un escala común, el cual se utilizó para ponderar la información base como pendiente, geología, geomorfología, cobertura y uso actual de tierras y la precipitación para obtener el mapa de peligros; de la misma forma para el análisis de la vulnerabilidad en la dimensión social, económica y ambiental, obteniendo como producto el mapa de riesgos por movimientos en masa de la subcuenca del río Ancash en un nivel alto. Las áreas caracterizadas con los niveles de riesgo identificado, son acompañados con una propuesta o medidas estructurales y no estructurales para su prevención y reducción del riesgo según sea el estado actual que presenten, buscando garantizar el desarrollo racional de las actividades socioeconómicas y ambientales de la población.

Palabras clave: riesgo, movimiento, masa

\section{ABSTRACT}

The sub-basin of the Ancash River located east of the city of Yungay has physically very varied characteristics expressed in geological, geomorphological and hydrological aspects. The increase in the frequency and intensity of extreme climatic phenomena, as a result of the variation in atmospheric conditions known as climate change, has triggered the occurrence 
of events such as mass movements. There are few national works in relation to the analysis of detonating factors (slope, geology, geomorphology and land use) and triggers (precipitation) to assess risks by mass movements with a basin approach, most of these works have been carried out in Experimental well-instrumented basins, but very few of these studies have been carried out taking the climatic characteristics as the anomalies in rainfall, one of the variables being the "E1 Niño" phenomenon that generates disastrous impacts on the population and their livelihoods.

The study carried out with the methodology of "Process of Hierarchical Analysis" (PAJ) that allows quantitative and qualitative criteria to be measured through a common scale, which was used to weight the base information such as slope, geology, geomorphology, coverage and current use of land and precipitation to obtain the hazard map; in the same way for the analysis of the vulnerability in the social, economic and environmental dimension, that allows quantitative and qualitative criteria to be measured through a common scale, which was used to weight the base information such as slope, geology, geomorphology, coverage and current use of land and precipitation to obtain the hazard map; in the same way for the analysis of the vulnerability in the social, economic and environmental dimension, obtaining as a product the map of risks by mass movements of the sub-basin of the Ancash river in a high level. The areas characterized by the levels of risk identified are accompanied by a proposal or structural and non-structural measures for their prevention and risk reduction according to the current state they present, seeking to guarantee the rational development of the socioeconomic and environmental activities of the population.

keyword: risk, movement, mass

${ }^{1}$ Universidad Nacional Santiago Antunez de Mayolo. ${ }^{a}$ Ing. Ambiental. ${ }^{b}$ Lic. En matemática 


\section{INTRODUCCIÓN}

La Cordillera Blanca, localizada en la región Ancash en la zona central y occidental del país, por sus características geológicas, sismotectónicas y climáticas, posee un alto índice de ocurrencia de eventos desastrosos (movimientos en masa detonados por sismos y lluvias (INGEMMET, 2016); la subcuenca del río Ancash tiene en la cabecera el nevado Huandoy perteneciente a la cadena montañosa de la Cordillera Blanca. Los fenómenos de remoción en masa son procesos de transporte de material definidos como procesos de 'movilización lenta o rápida de determinado volumen de suelo, roca o ambos, en diversas proporciones, generados por una serie de factores' (Hauser, 1993). Estos movimientos tienen carácter descendente ya que están fundamentalmente controlados por la gravedad (Cruden, 1991).

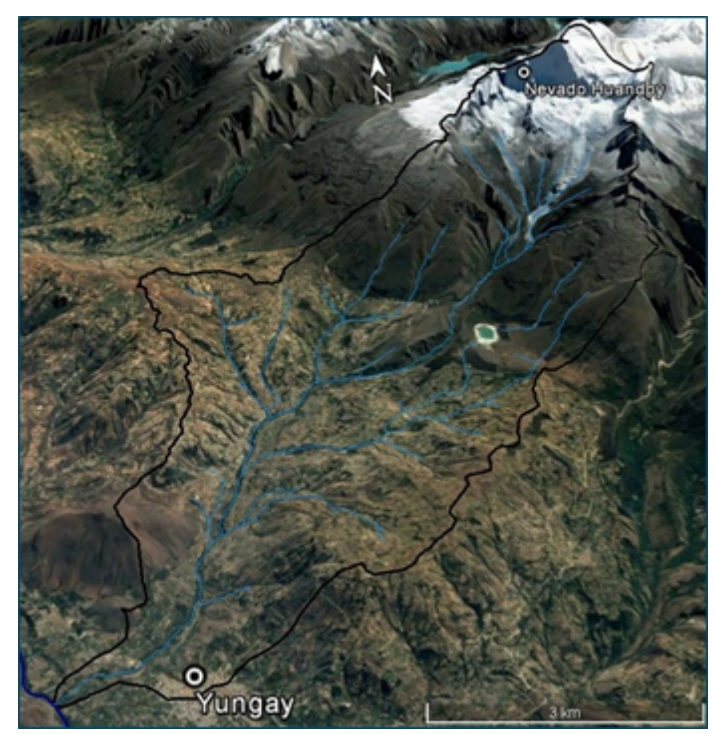

Figura 1. Ámbito de estudios

En la presente investigación se estimó el riesgo por movimientos en masa en la subcuenca del río Ancash, en el ámbito de la quebrada Ancash, distrito y provincia de Yungay. Se realizó el análisis y evaluación del peligro, análisis de la vulnerabilidad, la estimación del riesgo. Se proponen las medidas de control para mitigar los daños causados por los movimientos en masa. Si bien es cierto que existe una serie de factores condicionantes para el desarrollo de los eventos que deben ser identificados $\mathrm{y}$ estudiados, es asimismo de crucial importancia identificar el factor que se encarga de desencadenarlo. Entre los principales factores desencadenantes de estos fenómenos se encuentran los sismos, responsables principalmente de deslizamientos y caídas, y lluvias intensas, que generan en su mayoría flujos y deslizamientos, si bien ambos factores son capaces de desencadenar todos los tipos de remociones en masa (Lara, 2008). Los factores condicionantes son parámetros propios del ámbito geográfico de estudio, el cual contribuye de manera favorable o no al desarrollo del fenómeno de origen natural (magnitud e intensidad), así como su distribución espacial (Centro Nacional de Estimación, Prevención y Reducción del Riesgo de Desastres, 2013), se consideró la geología, geomorfología, pendiente y cobertura y uso actual de tierras; y como factor desencadenante se consideró la precipitación. El objetivo general fue estimar los niveles de riesgo por movimientos en masa en la subcuenca del 
del río Ancash. Los objetivos específicos fueron: Identificar y caracterizar los peligros por movimientos en masa en la subcuenca del río Ancash; Determinar los niveles de peligro por movimientos en masa en la subcuenca del río Ancash; Analizar la vulnerabilidad por movimiento en masa en la subcuenca del río Ancash; Estimar el riesgo por movimientos en masa en la subcuenca del río Ancash; Elaborar el mapa de riesgo por movimientos en masa en la subcuenca del río Ancash y Proponer medidas de control para mitigar los daños causados por los peligros de movimientos en masa en la subcuenca del río Ancash.

\section{MATERIALES Y MÉTODOS}

\section{Materiales}

\section{Imágenes digitales ráster}

Se utilizó un Modelo Digital de elevación de $12.5 \mathrm{~m}$ de resolución perteneciente al satélite de observación japonés ALOS que fue lanzado en 2006 por la Agencia Espacial Japonesa (JAXA), Las características de las imágenes ALOS AVNIR 2 las hacen adecuadas para estudios que requieren alto nivel de detalle, en áreas como estudios de uso y cobertura terrestre, manejo de recursos renovables y no renovables y monitoreo de desastres (United Nations Office on Drugs and Crime, 2017).

También se utilizaron imágenes satelitales de Google Earth del mes de junio de 2017, e imágenes y las imágenes satelitales de Bing Maps Aerial del año 2017, ambos descargados y corregidos geométricamente para su uso.

\section{Cartografía digital vectorial}

Para el mapa geológico se utilizaron la información vectorial (SHAPEFILE), del Instituto Geológico, Minero y Metalúrgico - INGEMMET, información descargado del portal web a una escala de 1: 50000 en coordenadas UTM-WGS84, los cuadrantes 19-h1 y 19-h4.

Para el mapa geomorfológico se tomo del estudio de "Peligros Geológicos por procesos glaciares en la cuenca del río Santa", que tiene como producto el mapa 5 "Geomorfología de la Cordillera Blanca" a una escala de 1: 200 000. Dicha información se digitalizó el área de estudio realizándose el ajuste correspondiente a la escala de trabajo.

\section{Cobertura y uso actual de tierras}

Para el cobertura y uso actual de tierras y cobertura vegetal se utilizó las imágenes satelitales de Google Earth y Bing Maps Aerial, que fueron digitalizados de acuerdo al uso y contrastadas in situ.

La literatura sobre el proyecto de investigación del tamarindo en el Perú nos puede revelar que no hay antecedentes sobre el tema en cuestión o que no son aplicables al contexto en el cual habrá de desarrollarse el estudio, ya que se tendrá que adecuar material de estudio cercano a nuestra investigación para fundamentar el trabajo de investigación.

\section{Análisis hidrometeorológico}

Los registros de precipitación se obtuvieron de las Estaciones Meteorológicas del Centro de 
Investigación Ambiental para el Desarrollo - CIAD de la Universidad Nacional Santiago Antúnez de Mayolo y de la Unidad de Glaciología y Recursos Hídricos de la Autoridad Nacional del Agua-ANA.

\section{Método multicriterio}

\section{Proceso de Análisis Jerárquico (PAJ)}

El Proceso de Jerarquía Analítica es un modelo para Toma de Decisiones, que fue desarrollado en el año de 1980, por el profesor Thomas Saaty, de la Universidad de Pittsburgh y es considera como una técnica multicriterio y multiatributo. La mecánica de aplicación es relativamente simple: 1) descomponer el problema; 2) realizar juicios comparativos; y 3) sintetizar los resultados(Morales, 2011). Utilizando la Tabla propuesta por Saaty y Vargas (1991) para la asignación de importancias o preferencias se puede asignar un puntaje de importancia relativa por parejas de indicadores, teniendo como referencia qué tanto, en forma comparativa, cada indicador refleja el aspecto que se desea representar.

Tabla 1. Escala de Saaty

\begin{tabular}{|c|c|c|}
\hline $\begin{array}{c}\text { ESCALA } \\
\text { NUMERICA }\end{array}$ & ESCALA VERBAL & EXPLICACIÓN \\
\hline 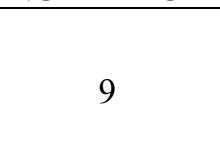 & $\begin{array}{l}\text { Absolutamente o } \\
\text { muchísimo más } \\
\text { importante que ... }\end{array}$ & $\begin{array}{l}\text { Al comparar un elemento con otro el } \\
\text { primero se considera absolutamente o } \\
\text { muchísimo más importante que el } \\
\text { segundo. }\end{array}$ \\
\hline 7 & $\begin{array}{l}\text { Mucho más } \\
\text { importante o } \\
\text { preferido que ... }\end{array}$ & $\begin{array}{l}\text { Al comparar un elemento con otro el } \\
\text { primero se considera absolutamente o } \\
\text { muchisimo más importante o preferido } \\
\text { que el segundo }\end{array}$ \\
\hline 5 & $\begin{array}{l}\text { Mas importante o } \\
\text { prefe rido que... }\end{array}$ & $\begin{array}{l}\text { Al comparar un elemento con otro el } \\
\text { primero se considera más importante o } \\
\text { preferido que el segundo }\end{array}$ \\
\hline 3 & $\begin{array}{l}\text { Ligeramente más } \\
\text { importante o } \\
\text { preferido que ... }\end{array}$ & $\begin{array}{l}\text { Al comparar un elemento con otro, el } \\
\text { primero es ligeramente más } \\
\text { importante o preferido que el segundo }\end{array}$ \\
\hline 1 & Igual o diferente a ... & $\begin{array}{l}\text { Al comparar un elemento con otro, hay } \\
\text { indiferencia entre ellos }\end{array}$ \\
\hline $1 / 3$ & $\begin{array}{l}\text { Ligeramente menos } \\
\text { importante o } \\
\text { preferido que ... }\end{array}$ & $\begin{array}{l}\text { Al comparar un elemento con otro, el } \\
\text { primero se considera ligeramente } \\
\text { menos importante o preferido que el } \\
\text { segundo }\end{array}$ \\
\hline $1 / 5$ & $\begin{array}{l}\text { Menos importante o } \\
\text { preferido que ... }\end{array}$ & $\begin{array}{l}\text { Al comparar un elemento con otro, el } \\
\text { primero se considera menos } \\
\text { importante o preferido que el segundo. }\end{array}$ \\
\hline $1 / 7$ & $\begin{array}{l}\text { Mucho menos } \\
\text { importante o } \\
\text { preferido que ... }\end{array}$ & $\begin{array}{l}\text { Al comparar un elemento con otro, el } \\
\text { primero se considera mucho menos } \\
\text { importante o preferido que el segundo }\end{array}$ \\
\hline $1 / 9$ & $\begin{array}{l}\text { Absolutamente o } \\
\text { muchísimo }\end{array}$ & $\begin{array}{l}\text { Al comparar un elemento con otro el } \\
\text { primero se considera absolutamente o } \\
\text { muchísimo más importante que el } \\
\text { segundo }\end{array}$ \\
\hline $2,4,6,8$ & \multicolumn{2}{|c|}{$\begin{array}{l}\text { Valores intermedios entre dos juicios adyacentes, que se } \\
\text { emplean cuando es necesario un término medio entre dos de } \\
\text { las intensidades anteriores }\end{array}$} \\
\hline
\end{tabular}

Fuente: Saaty (1980) 
Desarrollo matemático de la metodología de Proceso de Análisis Jerárquico (PAJ)

Para la ponderación de los criterios, sub criterios y descriptores se utilizó el Proceso de Análisis Jerárquico el cual es un método multicriterio que permite incorporar criterios cuantitativos (infraestructura expuesta, pérdidas humanas, económicas, etc.) y cualitativos (programas de capacitación, creación y/o aplicación de la normatividad, etc.) que son considerados en la Gestión del Riesgo de Desastres. La matriz que se forma es una matriz cuadrada es decir el mismo número de filas y columnas (Centro Nacional de Estimación, Prevención y Reducción del Riesgo de Desastres, 2015).

La notación matemática seria: $\boldsymbol{A}=\boldsymbol{A}_{\boldsymbol{i} \boldsymbol{j}}$

Para el cálculo de los pesos ponderados:

Primero: se construye la matriz de comparaciones pareadas, el que mostraría la comparación entre criterios, sub criterios y/o descriptores según el caso de interés. En el caso de ponderación de criterios esta matriz nos permite determinar la importancia de un criterio respecto a otro, lo que nos servirá posteriormente para la ponderación de criterios.

$$
A=\left(\begin{array}{cccc}
1 & a_{12} & \ldots & a_{1 n} \\
a_{21} & 1 & \ldots & a_{2 n} \\
\ldots & \ldots & \ldots & \ldots \\
\ldots & \ldots & \ldots & \ldots \\
\ldots & \ldots & \ldots & \ldots \\
a_{n 1} & a_{n 2} & \ldots & 1
\end{array}\right)
$$

Sumamos verticalmente los elementos de cada columna. Así se obtienen los valores:

$$
\boldsymbol{v} \mathbf{1}, \boldsymbol{v} \mathbf{2}, \ldots \boldsymbol{v} \boldsymbol{n}=\sum_{i=1}^{n} a_{i}
$$

Segundo: Construimos la matriz de comparaciones normalizada. El cual se obtiene de dividir cada elemento de matriz entre la suma obtenida, para conseguir:

$$
A_{\text {NORMALIZADA }}=\left(\begin{array}{cccc}
1 / v 1 & a_{12 / v 2} & \ldots & a_{1 n / v n} \\
a_{21 / v 1} & 1 / v 2 & \ldots & a_{2 n / v n} \\
\ldots & \ldots & \ldots & \ldots \\
\ldots & \ldots & \ldots & \ldots \\
\ldots & \ldots & \ldots & \ldots \\
a_{n 1 / v 1} & a_{n 2 / v 2} & \ldots & 1 / v n
\end{array}\right)
$$

Tercero: El siguiente paso consiste en obtener el vector prioridad el cual nos mostrará los pesos ponderados de cada criterio a partir de la matriz normalizada. Para ello se calcula el vector columna:

$$
\mathbf{p}=\left(\begin{array}{c}
\frac{1}{n} \sum_{j=1}^{n} a_{1 j} \\
\frac{1}{n} \sum_{j=1}^{n} a_{2 j} \\
\cdots \cdots \\
\cdots: \\
\frac{1}{n} \sum_{j=1}^{n} a_{n j}
\end{array}\right)
$$

Y se obtiene el vector de prioridades de los criterios:

$$
\mathbf{p}=\left(\begin{array}{c}
\mathrm{p}_{\mathrm{c} 11} \\
\mathrm{p}_{\mathrm{c} 12} \\
\cdots \\
\cdots \\
\cdots \\
\mathrm{p}_{c 1 n}
\end{array}\right)
$$

Se debe indicar que la suma de los elementos del vector prioridad debe ser iguala 1 .

$$
\sum_{i=1}^{n} \mathrm{p}_{c 1 i}=p_{c 11}+p_{c 12}+\ldots+p_{c 1 n}=1
$$




\section{Para el cálculo de la Relación de Consistencia (RC)}

Se pasa a la verificación de la posible existencia de consistencia entre los juicios expresados.

Primero: Multiplicar cada valor de la primera columna de la matriz de comparación pareada por la prioridad relativa del primer elemento que se considera y así sucesivamente. Se deben sumar los valores sobre las filas para obtener un vector de valores, denominado Vector Suma Ponderada (VSP).

$\left(\begin{array}{cccc}1 & a_{12} & \ldots & a_{1 n} \\ a_{21} & 1 & \ldots & a_{2 n} \\ \ldots & \ldots & \ldots & \ldots \\ \ldots & \ldots & \ldots & \ldots \\ \ldots & \ldots & \ldots & \ldots \\ a_{n 1} & a_{n 2} & \ldots & 1\end{array}\right) \times\left(\begin{array}{c}\mathrm{p}_{\mathrm{c} 11} \\ \mathrm{p}_{\mathrm{c} 12} \\ \ldots \\ \ldots \\ \ldots \\ \mathrm{p}_{c 1 n}\end{array}\right)=\left(\begin{array}{c}\boldsymbol{V S P}_{11} \\ \boldsymbol{V} \boldsymbol{S} \boldsymbol{P}_{12} \\ \ldots \\ \ldots \\ \ldots \\ \boldsymbol{V} \boldsymbol{S} \boldsymbol{P}_{1 n}\end{array}\right)$

Segundo: Dividir los elementos del vector de suma ponderada entre el correspondiente valor de prioridad para cada uno de los criterios:

$$
\begin{aligned}
& V S P_{11} / p_{C 11}=\lambda_{1} \\
& V S P_{12} / p_{C 12}=\lambda_{2} \\
& \text {..... } \\
& \operatorname{VSP}_{1 n} / \boldsymbol{p}_{C 1 n}=\lambda_{n}
\end{aligned}
$$

Tercero: Posteriormente se determina la lambda máxima $\lambda$ max

$$
\lambda_{\max }=\left(\lambda_{1}+\lambda_{12}+\cdots+\lambda_{n}\right) / n
$$

Esto nos permite hallar el índice de consistencia

Cuarto: Calcular el Índice de Consistencia (IC):

$$
I C=\left(\lambda_{\max }-\boldsymbol{n}\right) /(\boldsymbol{n}-\mathbf{1})
$$

Esto nos permite hallar la relación de consistencia de la matriz para verificar si las decisiones fueron adecuadas.

Quinto: Determinar la Relación de Consistencia (RC);

$$
R C=I C / I A
$$

Donde IA es el Índice Aleatorio de una Matriz de Comparaciones Pareadas, generada, como su nombre sugiere, de forma aleatoria. Los valores del Índice Aleatorio para los diferentes "n", obtenidos mediante la simulación de 100,000 matrices (Aguarón, 2003), son:

Tabla 2. Valores del índice aleatorio para los diferentes " $n$ "

\begin{tabular}{ccccccccccccccc}
\hline $\mathbf{N}$ & 3 & 4 & 5 & 6 & 7 & 8 & 9 & 10 & 11 & 12 & 13 & 14 & 15 & 16 \\
\hline $\mathbf{I}$ & 0.5 & 0.8 & 1.1 & 1.2 & 1.3 & 1.4 & 1.4 & 1.4 & 1.5 & 1.5 & 1.5 & 1.5 & 1.5 & 1.5 \\
$\mathbf{A}$ & 25 & 82 & 15 & 52 & 41 & 04 & 52 & 84 & 13 & 35 & 55 & 70 & 83 & 95 \\
\hline
\end{tabular}

\section{RESULTADOS}

\section{Análisis y evaluación del peligro}

Se identificaron 32 sectores críticos por movimientos en masa en la subcuenca de la quebrada Ancash, caracterizando de la siguiente manera: 13 por flujo de detritos, 5 por deslizamiento, 4 por caída de rocas, 8 por erosión de laderas y 2 por movimientos complejos. 


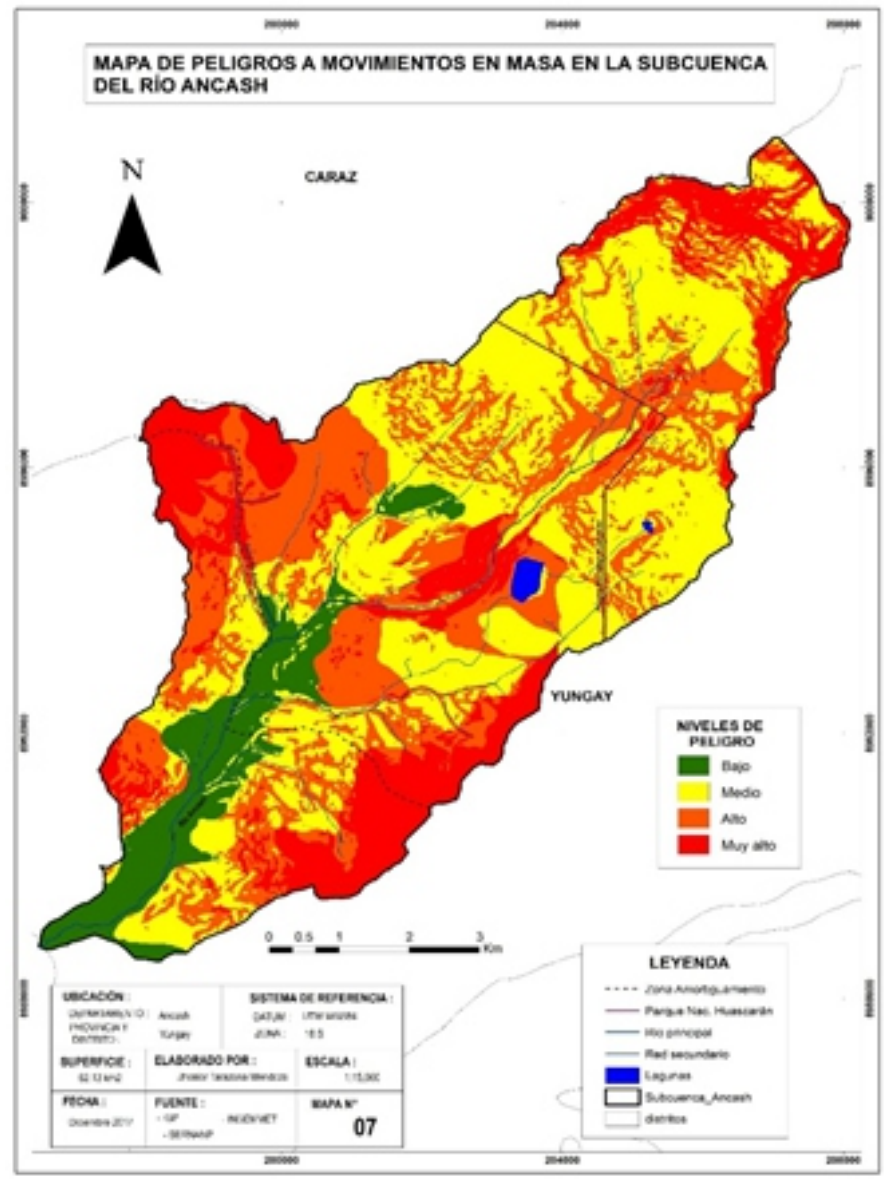

Figura 2. Mapa de Peligro

Tabla 3. Niveles de peligro

\section{NIVEL \\ DE DESCRIPCIÓN RANGOS \\ PELIGRO}

\begin{tabular}{ll}
\hline & Litología del grupo \\
& Gollarisquizga. Con pendiente \\
& superior a $35^{\circ}$. Geomorfología \\
Peligro & predominante de depósitos \\
Muy Alto & $\begin{array}{l}\text { coluviales y morrenas. En suelos } \\
\text { sin cobertura vegetal. }\end{array}$ \\
& Precipitaciones superiores 930 \\
& $\mathrm{~mm}$.
\end{tabular}

Los niveles de peligro por movimientos en masa se estratificaron en nivel muy alto, medio y bajo; de los cuales el valor del peligro es $\mathbf{0 . 2 8 1}$ indicando que el área de estudio tiene una peligrosidad alta por movimientos en masa.

\section{ANÁLISIS DE LA VULNERABILIDAD}

Los elementos vulnerables analizados en las dimensiones sociales, económicas y ambiental, obteniendo el valor de 0.224 , indicando que la vulnerabilidad es alta para la subcuenca del río Ancash. 


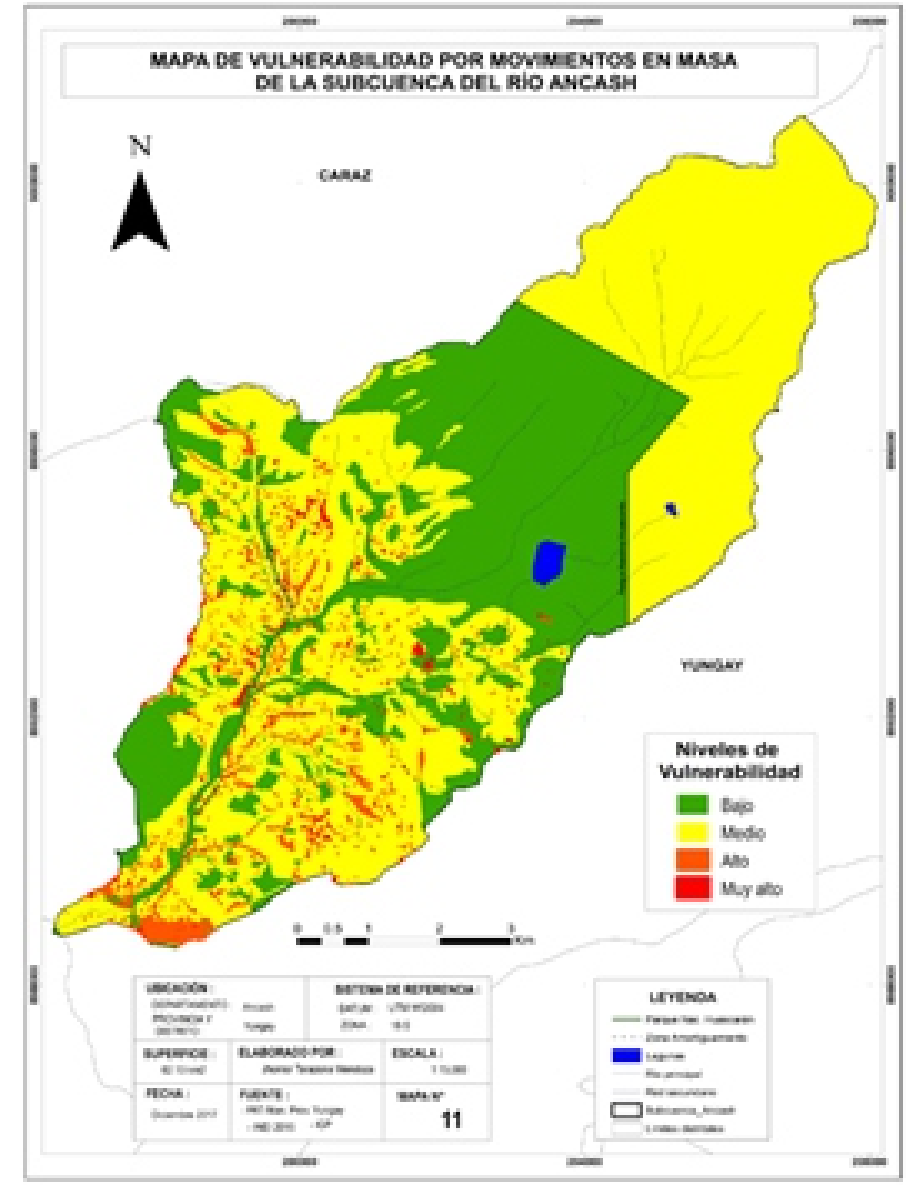

Figura 3. Mapa de vulnerabilidad

\section{ESTIMACIÓN DEL RIESGO POR MOVIMIENTOS EN MASA}

Para la estimación del riesgo se empleó la fórmula:

$$
\text { Riesgo }=\text { Peligro } x \text { Vulnerabilidad }
$$

Tabla 5. Niveles de riesgo

\begin{tabular}{ccc}
\hline \multicolumn{3}{c}{ Riesgo por movimientos en masa } \\
\hline Peligro & Vulnerabilidad & Valor \\
0.281 & 0.224 & $\mathbf{0 . 0 6 3}$ \\
\hline
\end{tabular}


Tabla 4. Niveles de vulnerabilidad

\begin{tabular}{|c|c|c|}
\hline $\begin{array}{c}\text { Nivel de } \\
\text { vulnerabilidad }\end{array}$ & Descripción & Rangos \\
\hline 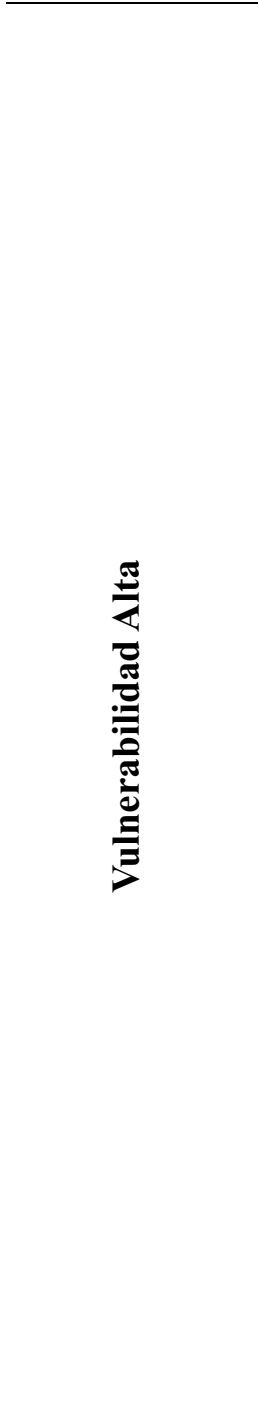 & $\begin{array}{l}\text { Según el grupo etario, de } 5 \text { a } 12 \text { años y de } 60 \text { a } \\
65 \text { años, servicio educativo y servicios de salud } \\
\text { expuestos de } 50 \text { a } 75 \% \text {, con material de } \\
\text { construcción de adobe y tapia, con estado de } \\
\text { conservación de la edificación que no reciben } \\
\text { mantenimiento, con pendiente de } 30 \text { a } 50 \% \text {, con } \\
\text { viviendas de } 3 \text { pisos, incumplimiento de } \\
\text { procedimientos de construcción de } 60 \text { a } 80 \% \text {, } \\
\text { con población escasamente capacitado, poco } \\
\text { conocimiento de escaso conocimiento sob re los } \\
\text { desastres, no existe soporte legal sobre la gestión } \\
\text { del riesgo de desastres, con actitud escasamente } \\
\text { previsora frente al riesgo, escasa difusión de } \\
\text { temas en gestión de riesgo, las viviendas están } \\
\text { localizadas cerca, los servicios de saneamiento } \\
\text { están expuestos de } 50 \text { a } 75 \% \text {, con área agrícola } \\
\text { de } 50 \text { a } 75 \% \text { expuesto, con antigüedad de las } \\
\text { edificaciones de } 30 \text { a } 40 \text { años, con bajo acceso o } \\
\text { poca permanencia en un puesto de trabajo, con } \\
\text { ingreso mensual de } 149 \text { a } 264 \text { soles, La población } \\
\text { está escasamente capaci tada en temas } \\
\text { concernientes a Gestión de Riesgo, Tierras } \\
\text { dedicadas a cultivos de pan llevar, Deforestación } \\
\text { agravada, uso indiscriminado de suelos y } \\
\text { sobrepastoreo, prácticas de consumo poblacional } \\
\text { inadecuados en riego, zonas medianamente } \\
\text { fracturada, los ce ntros ubicados medianamente } \\
\text { cerca, poco conocimiento de la normativas } \\
\text { ambientales, algunos pobladores poseen y aplica } \\
\text { sus conocimientos ancestrales para explotar de } \\
\text { manera sostenible sus recursos naturales. }\end{array}$ & 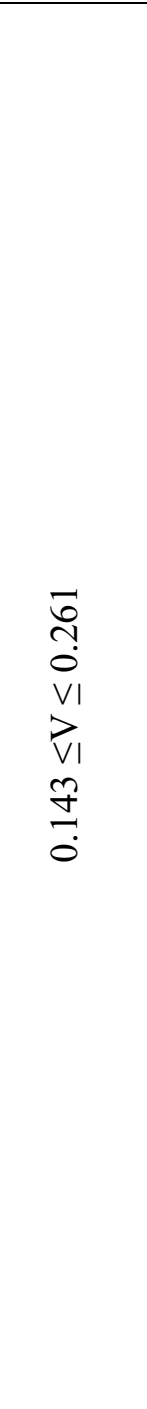 \\
\hline
\end{tabular}

De relacionar el peligro y la vulnerabilidad se obtiene el valor de 0.063 , el cual se encuentra en el intervalo $0.022 \leq 0.072$, correspondiente a un nivel de Riesgo Alto por movimientos en masa en la subcuenca del río Ancash.

Tabla 6. Niveles de riesgo

\begin{tabular}{ccc}
\hline Nivel del Riesgo & Rango & Color \\
\hline Riesgo Muy Alto & $0.072 \leq 0.192$ & \\
Riesgo Alto & $0.022 \leq 0.072$ & \\
Riesgo Medio & $0.007 \leq 0.022$ & \\
Riesgo Bajo & $0.002 \leq 0.007$ & \\
\hline
\end{tabular}




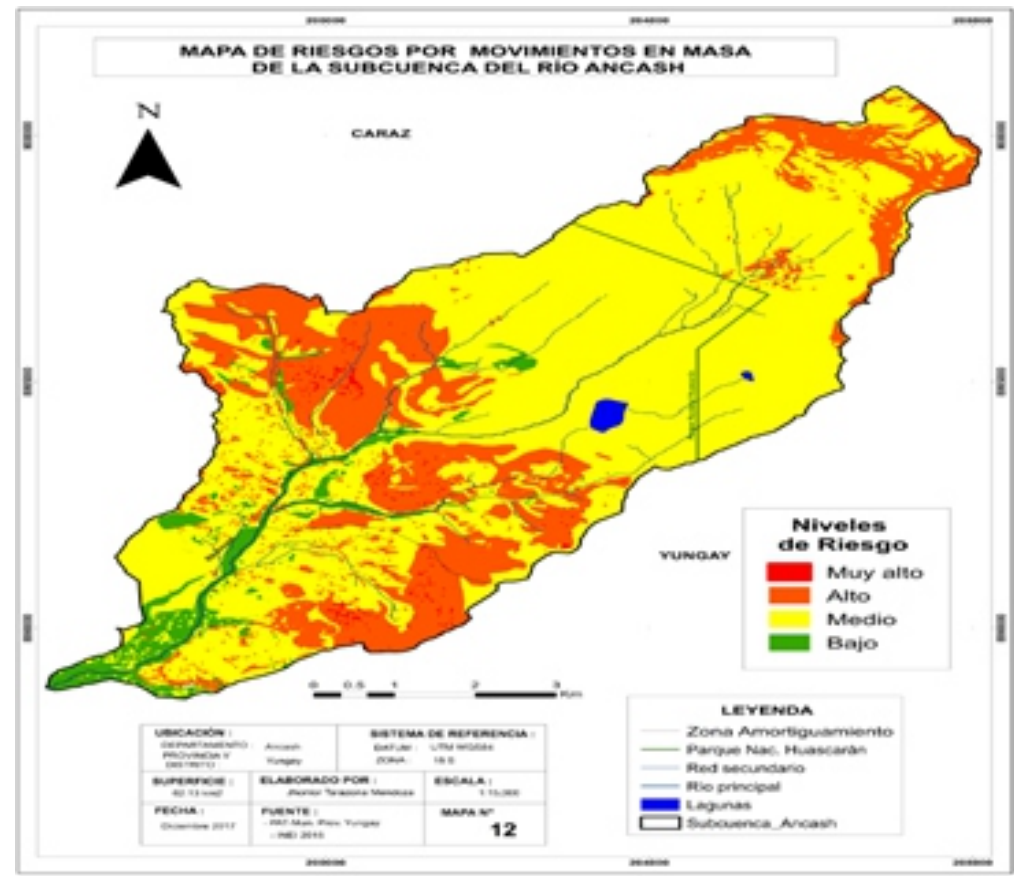

Figura 4. Mapa de riesgos por movimientos en masa

En caso no se controle el riesgo, los efectos por deslizamientos en la subcuenca del rio Ancash, generarían un costo de 17,100,807, ver el detalle en la Tabla $\mathrm{N}^{\circ} 5$.

Tabla 6. Probables efectos - costo por daños

\begin{tabular}{lc}
\hline Efectos probables & $\begin{array}{l}\text { Costo por } \\
\text { daños } \\
\text { probables }\end{array}$ \\
\hline Daños probables & $1,585,509$ \\
87 viviendas construidas con material de concreto & $12,594,629$ \\
1052 viviendas construidas con material precario & $1,819,473$ \\
10 instituciones educativas (publicas) construidos con & 485,474 \\
material precario & 32,702 \\
1 institución educativa construidas con material de concreto & 583,020 \\
5 iglesia & $\mathbf{1 7 , 1 0 0 , 8 0 7}$ \\
\hline Total Km de vías vecinales carrozables & \\
\hline
\end{tabular}

Fuente: Elaboración propia sobre los datos proporcionados por INEI-2015 y CENEPRED

\section{CONCLUSIONES}

La susceptibilidad y peligro por movimientos en masa de la subcuenca del río Ancash analizado con factor detonante la precipitación se obtuvo el valor de 0.281 categorizado como peligro muy alto, principalmente por flujo de detritos y erosión de laderas. Las dimensiones de vulnerabilidad social, económica y 18 Rev. Tayacaja 1(2): $08-20,2018$ ISSN:2617-9156 ambiental fueron analizadas y procesadas, obteniéndose el valor de 0.224 , correspondiente a vulnerabilidad alta para la subcuenca del río Ancash.El riesgo por movimientos en masa en la subcuenca, resultado de la relación del peligro y vulnerabilidad, dio como resultado el valor de 0.063 , equivalente al nivel de riesgo alto. 
En subcuenca del río Ancash, el mapa de riesgos por movimientos en masa presenta 4 niveles de riesgo. Un área de $312.77 \mathrm{Ha}$. equivalente al 5.04\% del territorio presenta riesgo bajo, $4233.42 \mathrm{Ha}$. equivalente a $68.17 \%$ del territorio, presenta riesgo medio, $1621.14 \mathrm{Ha}$. equivalente a $26.11 \%$ del territorio, presenta riesgo alto y $42.44 \mathrm{Ha}$. equivalente a $0.68 \%$ del territorio, se presenta como riesgo muy alto. La estimación del impacto por la materialización del riesgo en los niveles alto y muy alto, se generaría daños en 87 viviendas de material noble, en 1052 viviendas de material precario (adobe, tapial y piedra), en 10 instituciones educativas públicas de material precario, en 1 institución educativa de material noble, en 5 iglesias y en 23.71 kilómetros de vías vecinales carrozables, con un costo aproximado de S/. 17,100,807 Soles.

\section{RECOMENDACIONES}

Se recomienda realizar la evaluación del riesgo por sismo debido a que en la subcuenca del río Ancash se encuentra la falla geológica de la Cordillera Blanca.

Se recomienda realizar la evaluación del riesgo de los ecosistemas de montaña ya que la cabecera de la subcuenca del río Ancash es Área Natural Protegida (ANP) que pertenece al Parque Nacional de Huascarán. Incorporar a la metodología los factores desencadenantes propios del proceso del cambio climático como la desglaciación, lluvias intensas y el incremento de la temperatura.Realizar estudios similares para determinar escenarios futuros del cambio climático en la subcuenca del río Ancash y establecer medidas de adaptación.

\section{Propuesta de medidas de prevención y reducción de desastres}

Con el propósito de reducir el costo por la materialización del riesgo en la subcuenca del rio Ancash, se proponen las siguientes medidas:

\section{Medidas estructurales}

Construcción de muros de contención de vías de comunicación en los siguientes lugares:

- A la entrada d e Atma aproximadamente 230 metros lineales debido a que existe muchos deslizamientos en estaciones lluviosas.

- En Huincush, Huepes, Shacllaqueru y Cochapampa en las curvas que se encuentran en la parte baja de los humedales y ojos de agua con aproximadamente de 80 metros lineales de intervención.

- En Panash existen 2 punto críticos que afectan la carretera con deslizamiento es necesario la construcción de 55 metros lineales necesario intervenir.

- En Rayan y Shuyon se ha identificado 12 puntos críticos de cada una de 6 metros lineales necesarias de intervención.

- $\quad$ En tucuhuran hasta Marap existen 3 puntos críticos de 18 metros lineales en promedio de cada una donde es necesaria la intervención.

Forestación y reforestación en los siguientes sectores: umac shoc aproximadamente $7.3 \mathrm{Ha}$, en Churu Huarca aproximadamente $4.3 \mathrm{Ha}$, en la parte alta de Mazacpampa aproximadamente $5.5 \mathrm{Ha}$ y en Curicasha aproximadamente $9 \mathrm{Ha}$. Siendo 
recomendable con plantaciones de pino, aliso y ciprés por su capacidad adaptativa. Construcción de sistemas de drenaje aproximadamente $1.5 \mathrm{Km}$. en la parte alta de Huepesh debido a la alto deslizamiento y flujo de detritos que está afectando áreas agrícolas.

\section{Medidas no estructurales}

- Como municipalidad es necesario el desarrollo y fortalecimiento institucional con la implementación de su Centro de Operaciones de Emergencia y contar con profesionales especializados en gestión del riesgo de desastres como son los evaluadores de riesgo.

- Elaboración de planes de contingencia, planes de prevención y reducción del riesgo de desastres, planes de educación comunitario.

- Incorporación del mapa de riesgo por movimientos en masa en la gestión territorial para la adecuada toma de decisiones.

- Realizar capacitaciones para la reducción del riesgo por movimientos en masa.

- Prever la participación permanente de los comités de gestión del riesgo y desarrollar faenas para minimizar los impactos por peligro de movimientos en masa.

\section{REFERENCIAS BIBLIOGRÁFICAS}

Aguarón, J. a. (2003). The geometric consistency index:Approximated thresholds. European Journal of Operational Research.

Centro Nacional de Estimación, Prevención y Reducción del Riesgo de Desastres. (2013). Manual para la Evaluación de Riesgos Originados por Fenómenos Naturales. Lima.

Centro Nacional de Estimación,
Prevención y Reducción del Riesgo de Desastres. (2015). Manual para la Evaluación de Riesgos originados por Fenómenos Naturales (02 Versión). Lima-Perú: NEVA STUDIO SAC.

Corporación Andina de Fomento. (2000). El Fenómeno el NIño 1997-1998 Memorias, retos y soluciones (Vol. V). Lima, Perú: Corporación Andina de Fomento.

Cruden, D. (1991). simple definition of a Landslide. En Bulletin of the International Association of Engineering Geology (pág. 43).

El Instituto Geológico Minero y Metalúrgico. (2016). Peligros Geológicos por Procesos Glaciales, Cordillera Blanca Río Santa. Lima, Perú: INGEMMET.

Hauser, A. (1993). Remociones en masa en Chile. . Santiago: Servicio Nacional de Geología y Minería.

Lara, M. C. (2008). Remociones en Masa. Santiago.

Morales, F. J. (2011). Aplicación de métodos de toma de definición de prioridades en la gestión de la infraestructuras en San Luis de Potosí, México. Madrid.

United Nations Office on Drugs and Crime. (14 de Diciembre de 2017). Oficina de las Naciones Unidad contra la Droga y el Delito. Obtenido de http://www.biesimci.org/satelita 1/original/ALOS/ALOSindex.ht $\mathrm{ml}$

Villanueva, R. R. (2011). Los impactos del cambio climático en las funciones hidrológicas de la cuenca del río Santa. Huaraz, Ancash, Perú: Corporación Globalmark.

\section{CORRESPONDENCIA}

Jhonior Tarazona Mendoza haga1379@gmail.com 